Research Article

\title{
Synergistic Effects of Nanoemulsion and Deferiprone (1, 2 Dimethyl-3-Hydroxypyrid- 4-One) on Multi-Drug Resistant Acinetobacter baumannii
}

\author{
Karthikeyan Ramalingam ${ }^{1}$, Valerie Lee ${ }^{2}$ \\ ${ }^{1}$ School of Life Sciences, B.S. Abdur Rahman Crescent Institute of Science and Technology, Vandalur, Chennai, Tamil Nadu, 600048 \\ India. \\ ${ }^{2}$ Department of Comprehensive Dentistry, University of Texas Health at San Antonio, 7703 Floyd Curl Drive, San Antonio, Texas \\ 78229-3900, USA.
}

C Corresponding author. E-mail: karthikeyan.sls@crescent.education; hydrophis.07@gmail.com; Tel.: +91 44 22759223; +91 44 22751347; Mobile: +91 7502534668

Received: Mar. 26, 2019; Accepted: Jul. 13, 2019; Published: Jul. 23, 2019.

Citation: Karthikeyan Ramalingam, Valerie Lee, Synergistic Effects of Nanoemulsion and Deferiprone (1, 2 Dimethyl-3-Hydroxypyrid-4-One) on Multi-Drug Resistant Acinetobacter baumannii. Nano Biomed. Eng., 2019, II (3): 226-237.

DOI: 10.5101/nbe.v11i3.p226-237.

\begin{abstract}
Nosocomial infections caused by Acinetobacter baumannii (A. baumannii) are exceedingly difficult to treat, particularly in immune-compromised patients. Nanoemulsions (NEs) are a distinctive category of disinfectants that have wide range of bactericidal, fungicidal and antiviral properties by damaging their outer membranes. The inhibiting potential of iron chelators such as deferiprone (DF) used as remedial agent for different microbial groups. Combining new generation disinfectant NEs and the iron chelator DF is a logical approach to control of A. baumannii planktonic and biofilm infections. Present studies investigated the bactericidal efficacy of NE with DF by testing their minimum inhibitory concentration (MIC) and minimum bactericidal concentration (MBC) against four strains of $A$. baumannii. We analyzed adherence of the planktonic form both with and without deferiprone to determine possible enhancement effects. The mechanism of nanoemulsion with deferiprone (NE/DF) was quantified by LIVE/DEAD staining biofilm assay and scanning electron microscopy. DF does not affect the stability of the nanoemulsion and the combined forms show high stability. From MBC, $\mathrm{NE} / \mathrm{DF}$ showed enhanced activity up to 2- to 3.3-fold compared to nanoemulsion and deferiprone alone. Combined nanoemulsion with deferiprone showed higher adherence inhibition compared to the individual forms. From confocal imaging, the level of dead cell intensity was $84.9 \%$ to $96.6 \%$ in $\mathrm{NE} / \mathrm{DF}$ treated groups. Bacteria treated with NE, DF and NE/DF confirms morphological variations when compared to pili-bonded integrated control cells. We propose that biofilm destruction starts from pili removal followed by membrane destruction. These results leave a concrete evidence for the employment of nanoemulsion with deferiprone for the treatment of against drug resistant $A$. baumannii.
\end{abstract}

Keywords: Nanoemulsions; Deferiprone; Acinetobacter baumannii; Biofilm; Antimicrobial agent

\section{Introduction}

Nanoemulsions (NEs) are a distinct category of disinfectants formed by integration of an aqueous phase and an oily phase under high pressure. Nanoemulsions consist of identical population of 
oil droplets with diameters of 200-800 $\mathrm{nm}$ [20]. Nanoemulsions have been found to have a wide range of biocidal effect against bacteria, fungi and enveloped viruses, by disrupting their outer membranes [1-3]. The membranes' disruption properties have facilitated the damages in bacteria [4], fungi in the even dilutions of 1:1000 [2,5], and enveloped viruses [5, 6] are the nanoemulsions which are the non miscible combinations of water and soybean oil.

The inhibiting potential of iron chelators as remedial agent for different microbial groups has been proved successfully [7-9]. 1,2 dimethyl-3-hydroxypyrid4-one (Deferiprone) is an example of iron chelator. However, as new iron-chelators have been synthesized for managing applications of neurodegenerative or $\beta$-thalassemia $[10,11]$, antimicrobial activity by novel iron chelators should be re-evaluated. Antibacterial effects of the iron chelator deferiprone (DF) have not yet been tested against bacterial biofilms. Current available classes of antibiotics are no longer the treatment for multidrug-resistant bacteria (MDR) stains [12], iron chelators might provide an alternative treatment options, because it's already been thorough toxicology and animals' preclinical testing. Combining the new generation disinfectant NEs and the iron chelator DF is a logical approach to control planktonic and biofilm infection of MDR-bacterial pathogens such as Acinetobacter baumannii (A. baumannii). A. baumannii needs extremely complicated treatment because of its nosocomial behavior [13] and has also been liable for wound infections caused by military work force which often cause compromised immune system following polytrauma [14]. A. baumannii resistance is mainly because of its lower permeability of outer membrane against some of the antibiotics. In addition, these bacteria can effortlessly obtain and integrate genetic materials like plasmids, transposoable elements and integrons [15]. Infections due to such resistant microbes are associated with increased morbidity and mortality [16].

This paper investigated the antimicrobial efficacy of an EDTA-containing nanoemulsion and deferiprone by testing inhibitory and bactericidal concentration on four strains of $A$. baumannii. This paper examined the adherence inhibition of the planktonic $A$. baumannii with and without deferiprone to establish any synergistic behavior. The effectiveness of nanoemulsion and deferiprone was quantified by LIVE/DEAD staining biofilm assay and observation by scanning electron microscopy.

\section{Experimental Bacteria and growth conditions}

A. baumannii strains (ATTC BAA-1605, 17961, 19606, 19003) were purchased from ATCC (ATCC, Manassas, VA). The strain BAA-1605 was a multidrug resistant one isolated from the 2006 military personnel's sputum [17]. An A. baumannii strain 17961, 19606 and 19003 was cultured in sources of blood, urine and cerebrospinal fluid of patients, respectively. The media used for bacteria were nutrient broth and agar (BD, Franklin Lakes, NJ). For all the studies, cultures with optical density (OD) 0.3-0.4 at $490 \mathrm{~nm}\left(10^{7} \mathrm{CFU} / \mathrm{mL}\right)$ were used.

\section{Preparation and characterization of nanoemul- sion and deferiprone}

The o/w nanoemulsion consisted of ingredients of $25 \%$ soybean oil (v/v), surfactant Brij $30(10 \% \mathrm{v} / \mathrm{v})$, with $60 \mathrm{mM}$ EDTA $(65 \% \mathrm{v} / \mathrm{v})$ in deionized water. Emulsion was prepared using a microfluidizer (M-110L, Microfluidics, Newton, MA) with 2 runs at 20,000 PSI under room temperature. Laser electrophoresis light scattering system was used to measure droplet size and zeta potential (Zeta plus, Zeta Potential and Particle Size Analyzer, Brookhaven Instruments, Holtsville, NY). This nanoemulsion was used for all experiments. Deferiprone (Sigma Aldrich, St. Louis, MO) was added to the nanoemulsion at the concentration of 0.25 to $10 \mathrm{mM}$ (100 mM serially diluted for MIC and MBC study; 0.25 to $2 \mathrm{mM}$ used for adherence study; $10 \mathrm{mM}$ concentration for biofilm study). Being hydrophilic, deferiprone resided in the water phase of the emulsion.

\section{Determination of minimum inhibitory concentration (MIC) and minimum bactericidal concentration (MBC)}

Nanoemulsion, deferiprone and nanoemulsion in combination with deferiprone were serially diluted with sterile nutrient broth. $20 \mu \mathrm{L}$ of standardized bacterial culture $\left(10^{7} \mathrm{CFU} / \mathrm{mL}\right)$ was added to each well of the 96 well plates. It was then incubated at $37^{\circ} \mathrm{C}$ overnight and MIC was found. $100 \mu \mathrm{L}$ of culture broth from the wells which had no growth were plated onto nutrient agar and incubated overnight at $37{ }^{\circ} \mathrm{C}$ to determine MBC. The highest dilution that resulted in 99.9\% reduction in the bacterial cell number was noted as MBC. The negative control used contained sterile distilled water.

\section{Adherence assay}

Overnight bacterial cultures containing $10^{7} \mathrm{CFU} / \mathrm{mL}$ 
of Acinetobacter baumannii strains (ATTC BAA-1605, 17961, 19606, 19003) were added to different dilutions and concentrations of nanoemulsion (NE, 1000, 2500, 5000 and 7500), deferiprone (DF, 0.25, 0.50, 1 and 2 $\mathrm{mM}$ ) and combined NE/DF. Slanted glass coverslips $(20 \mathrm{~mm} \times 11 \mathrm{~mm})$ inserted into 24-well microtiter plates were used for this experiment. Plates were incubated at $37^{\circ} \mathrm{C}$ for $24 \mathrm{~h}$. The glass coverslips were transferred to new 24-well plates following incubation and fixation using methanol for $15 \mathrm{~min}$. Plates containing the glass coverslips were then air dried. All the wells were stained with $0.1 \%(\mathrm{w} / \mathrm{v})$ crystal violet for $5 \mathrm{~min}$. By placing the plate under running tap water, excess stain was removed. The plate was further air dried, and the bound dye was removed using 33\% (v/v) glacial acetic acid. $595 \mathrm{~nm}$ was used to measure the optical density of the solution (BioTek Instruments Inc, Winooski, VT). For each experimental condition, three independent experiments were performed. The negative controls' mean OD was subtracted from all other samples. Nanoemulsion of 1000-time dilution consisted of $25 \mathrm{mg}$ soybean oil, $10 \mathrm{mg}$ surfactant Brij 30 , and $11.39 \mathrm{mg}$ of EDTA.

\section{Live and dead biofilm assay}

For the biofilm viability test, $A$. baumannii strains (ATTC BAA-1605, 17961, 19606 and 19003) were grown on slanted glass coverslips inserted into 24-well microtiter plates. The plates were incubated aerobically for $72 \mathrm{~h}$ at $37{ }^{\circ} \mathrm{C}$. Unattached platonic bacterial cells were replaced by the same volume of fresh medium at every $12 \mathrm{~h}$ interval for the healthy biofilm formation. The glass coverslips with the grown biofilm were treated with 250 dilution of NE, $10 \mathrm{mM}$ of DF, or combined nanoemulsion with deferiprone (250 dilution $\mathrm{NE}$ and $10 \mathrm{mM} \mathrm{DF}$ ) for $1 \mathrm{~h}$. Following the treatment, the biofilm was stained with L 7012 LIVE/DEAD BacLight $^{\mathrm{TM}}$ Bacterial Viability Kit from Molecular Probes Inc. (Eugene, OR) as described by Neu and Lawrence [18]. The samples were immediately examined via Nikon ECLIPSE -TE200 with EZC1 (version 3.40) software (Nikon Instruments, Inc. Melville, New York) at excitation wavelengths of 488 and $543 \mathrm{~nm}$. For image analysis, a maximal projection of each image stack was built using the program Nikon imaging software NIS-Elements AR3.00, SP-4, Hotfix6 (Build 505), and the red and green projections were selected separately to measure their intensity. The resulting green and red intensities were analyzed for their statistical significance. Three separate experiments were done to confirm the experimental adequacy.
Nanoemulsion of 250-time dilution consisted of 100 $\mathrm{mg}$ soybean oil, $40 \mathrm{mg}$ surfactant Brij 30, and 45.59 $\mathrm{mg}$ of ethylenediaminetetraacetic acid (EDTA).

\section{Scanning electron microscopy (SEM) observation}

Biofilm formation as mentioned live-dead biofilm assay, for SEM observation. The biofilm was exposed with 250 dilution of $\mathrm{NE} ; 10 \mathrm{mM}$ of $\mathrm{DF}$ or combined nanoemulsion with deferiprone (250 dilution $\mathrm{NE}$ and $10 \mathrm{mM}$ DF) was added to the biofilms present on the glass coverslips for $1 \mathrm{~h}$, and then gently washed with sterile deionized water twice. Biofilm fixation on glass slides was carried out as per the method described by Araujo et al. [19]. In a graded series of cold ethanol/ water mixture $(50 \%, 70 \%, 80 \%, 90 \%, 95 \%$ and $100 \%$ of ethanol), the fixed biofilms were dehydrated for 10 min. Further 10 min dehydration was carried out with $100 \%$ ethanol twice. Biofilms present on the glass slides were treated with 1:1 ratio of ethanolhexamethyldisilazane (HMDS) (Electron Microscopy Sciences, Hatfield, PA) for $5 \mathrm{~min}$ and 100\% HMDS for $100 \mathrm{~min}$ with mild rocking. Biofilms were airdried following dehydration. Then biofilm specimens were covered with a gold-palladium (1:1) target using a Hummer 6.2 Sputter Coater (Anatech USA, Union City, CA). The samples were examined using SIGMA VP40 Scanning Electron Microscope (Carl Zeiss, Inc., Germany) in high vacuum mode at $2 \mathrm{kV}$.

\section{Activity of nanoemulsion and its components}

Live-dead biofilm assay was conducted for $A$. baumannii (ATTC BAA-1605) biofilm formation as mentioned. The glass coverslips with grown biofilm was treated with nanoemulsion (10\% Brij, 25\% soybean oil, $60 \mathrm{mM}$ EDTA and $65 \%$ water), or with the individual ingredients (10\% Brij, $25 \%$ soybean oil, and $60 \mathrm{mM}$ EDTA in deionized water) for $1 \mathrm{~h}$. Biofilms were staining, and the image analysis was carried out as described for live and dead biofilm assay.

\section{Statistical analysis}

The means and standard deviations were calculated. Two-way ANOVA (adherence and LIVE/DEAD staining assays) were used to determine the levels of significance $(p<0.05)$.

\section{Results and Discussion \\ Preparation and characterization of nanoemulsion and deferiprone}

The nanoemulsion had the appearance and 
consistency of whole milk (Fig 1 inserts). Particle size distributions of microfluidized nanoemulsion were $295.3 \mathrm{~nm}$ with 0.065 polydispersity, $5.5 \mathrm{~nm}$ with 0.390 polydispersity for $10 \mathrm{mM}$ deferiprone, and $293.0 \mathrm{~nm}$ with 0.056 polydispersity for combined DF and NE. Zeta potential values showed a higher level of stability for nanoemulsion $(-61.80 \mathrm{mV}, \mathrm{pH} 8.18)$ and combined nanoemulsion with deferiprone (-54.54 mV, pH 7.38). Deferiprone showed a $0.00 \mathrm{mV}$ zeta potential with $\mathrm{pH}$ of 5.24, indicating that deferiprone alone was the least stable form (Fig. 1). Since deferiprone combined with nanoemulsion showed a reduction of zeta potential, the stability of the end product may not be affected.

\section{Determination of minimal inhibitory concentration (MIC) and minimum bactericidal concentration (MBC)}

Due to the highly turbid nature of nanoemulsion compared to bacterial culture, the MIC was not recorded. The MBC of combined nanoemulsion with deferiprone (NE/DF) for A. baumannii S1 (BAA-
1605) (320 dilution / 3.13mM), S2 (ATCC 17961) (213 dilution / $5.21 \mathrm{mM}$ ), S3 (ATCC 19606) (427 dilution / $2.6 \mathrm{mM}$ ) and S4 (ATCC 19003) (320 dilution / 3.13 $\mathrm{mM}$ ) were recorded. Higher MBCs were observed for S1 and S4 than for S2 and S3. Synergistic interaction of nanoemulsion and deferiprone resulted in increased activity up to 2- to 3.3-fold compared to either nanoemulsion or deferiprone (Fig. 2).

\section{Adherence assay}

The potential of nanoemulsion to act synergistically with deferiprone against A. baumannii during adherence was evaluated (Fig. 3). The inhibitory effect of NE/DF on adherence of cells to glass surfaces resulted in higher inhibition of the growing cells. ANOVA indicated that adhesion of all strains was significantly $(p<0.05)$ affected by nanoemulsion combined with deferiprone. Highest adherence was observed with A. baumannii grown with 7500 fold dilution $\mathrm{NE}(86.5 \%$ for $\mathrm{S} 3$ ) and $0.25 \mathrm{mM}$ of DF concentration $(88.8 \%$ for $\mathrm{S} 1)$. The combined form
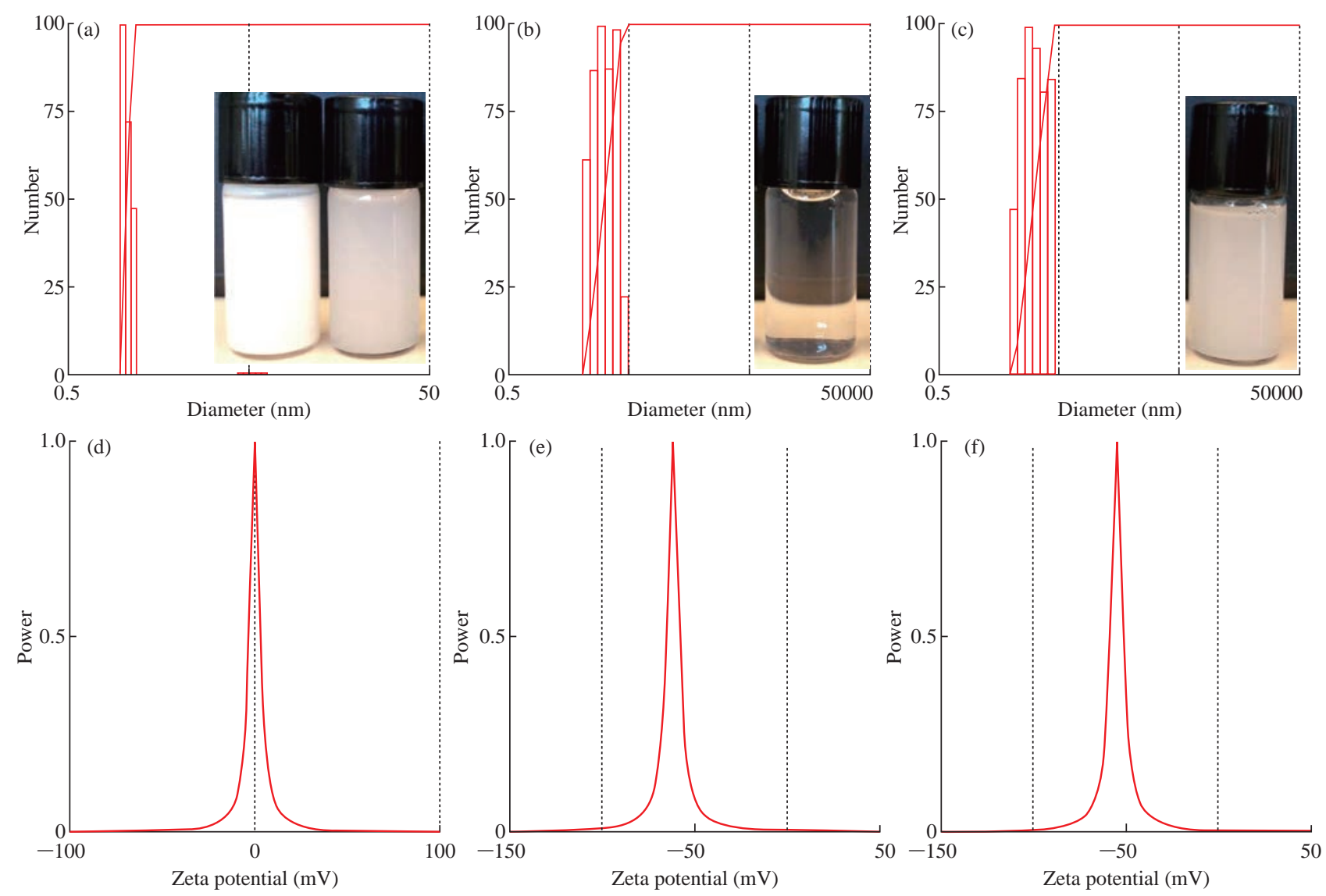

Fig. 1 Laser electrophoresis light scattering image showing (a)-(c) distribution of particle-size and (d)-(f) zeta potential. (a) and (d) Deferiprone; (b) and (e) Nanoemulsion; (c) and (f) Nanoemulsion with deferiprone. Inserts showing the appearance of nanoemulsion, deferiprone, and nanoemulsion with deferiprone. ((a) Undiluted nanoemulsion and 250-fold diluted nanoemulsion; (b) $10 \mathrm{mM}$ deferiprone; and (c) Nanoemulsion with deferiprone.) 


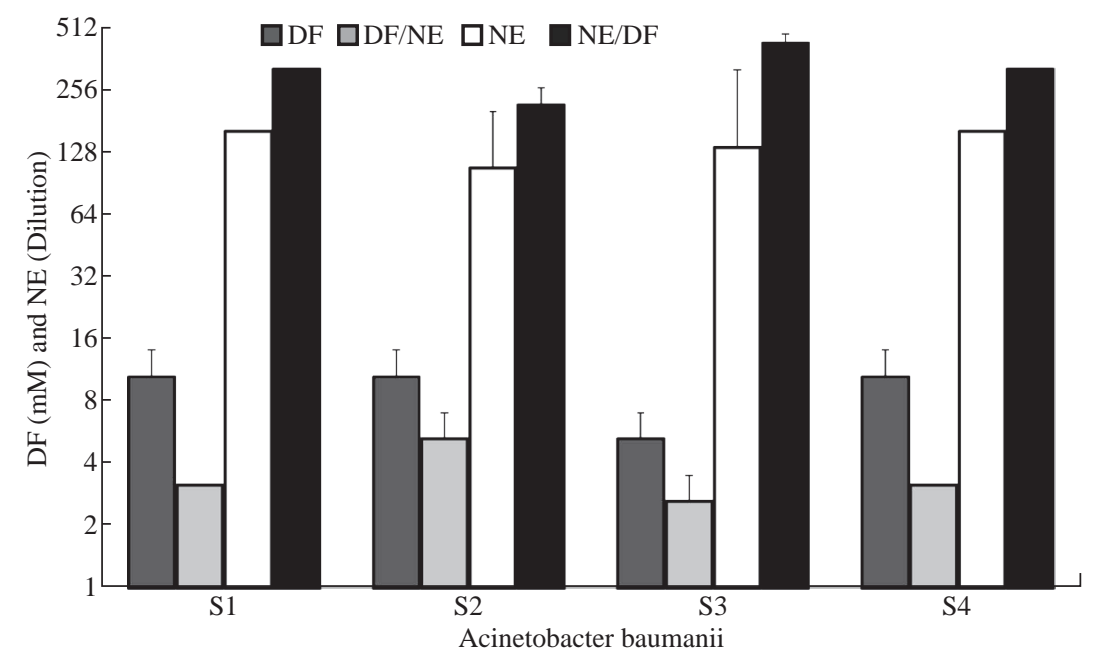

Fig. 2 Minimum bactericidal concentration of nanoemulsions (NE), deferiprone (DF), and nanoemulsion with deferiprone (NE/DF) on Acinetobacter baumannii strains. S1 BAA-1605; S2 ATCC-17961; S3 ATCC-19606; and S4 ATCC-19003.
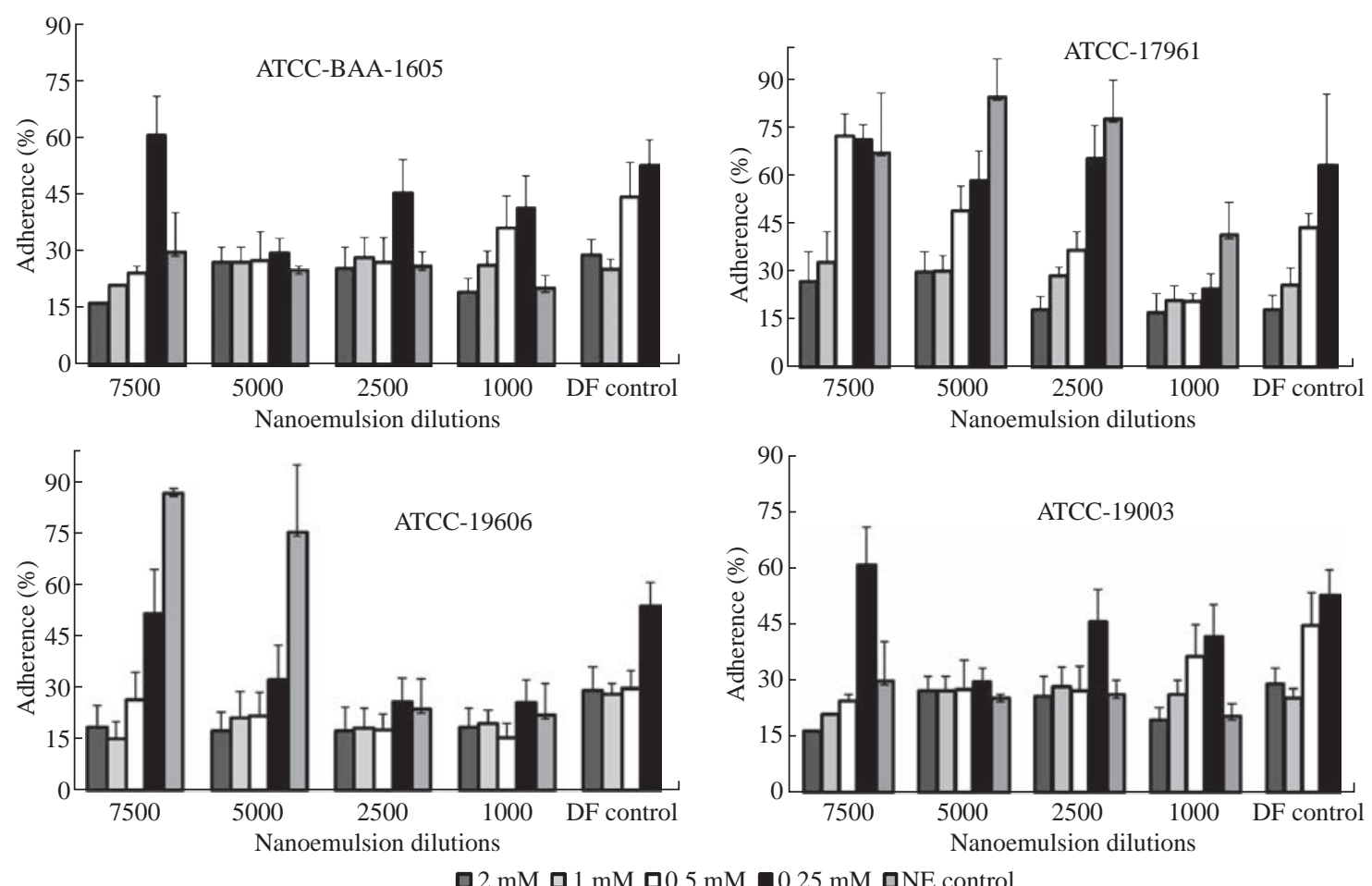

Fig. 3 Efficacy of nanoemulsion (NE), deferiprone (DF), and nanoemulsion with deferiprone (NE/DF) on adherence of S1 Acinetobacter baumannii strains (ATCC-BAA-1605; ATCC-17961; ATCC-19606; ATCC-19003).

of nanoemulsion with deferiprone (NE/DF) showed higher adherence inhibition compared to the individual ingredients, and lower dilution of nanoemulsion (1000 dilution); higher concentration deferiprone (2 $\mathrm{mM})$ showed higher inhibition of adhesion. Inhibition of adhesion by NE/DF ranged from S1 (21.5 to $83.7 \%$ ), S2 (27.9 to $82.9 \%)$, S3 (48.4 to $84.9 \%)$, and S4 (29.1 to $83.6 \%)$.

\section{Live and dead biofilm assay}

To establish the LIVE/DEAD patterns within control and treatment groups in the four stains of A. baumannii biofilm, biofilms grown on slanted glass cover slips $(20 \mathrm{~mm} \times 11 \mathrm{~mm})$ were placed into 24-well micro titer plates and stained with the LIVE/DEAD reagent (Fig. 4 \& 5). In the control groups, most of the biofilm stained "live" (green), whereas in the treatment (nanoemulsion with deferiprone) groups cells throughout the structure stained "dead" (red). SYTO 9 and propidium iodide are the two fluorescent nucleic acid stains present in LIVE/DEAD stain. SYTO 9 was used to enumerate "live" cells as it could pass through cells under every 

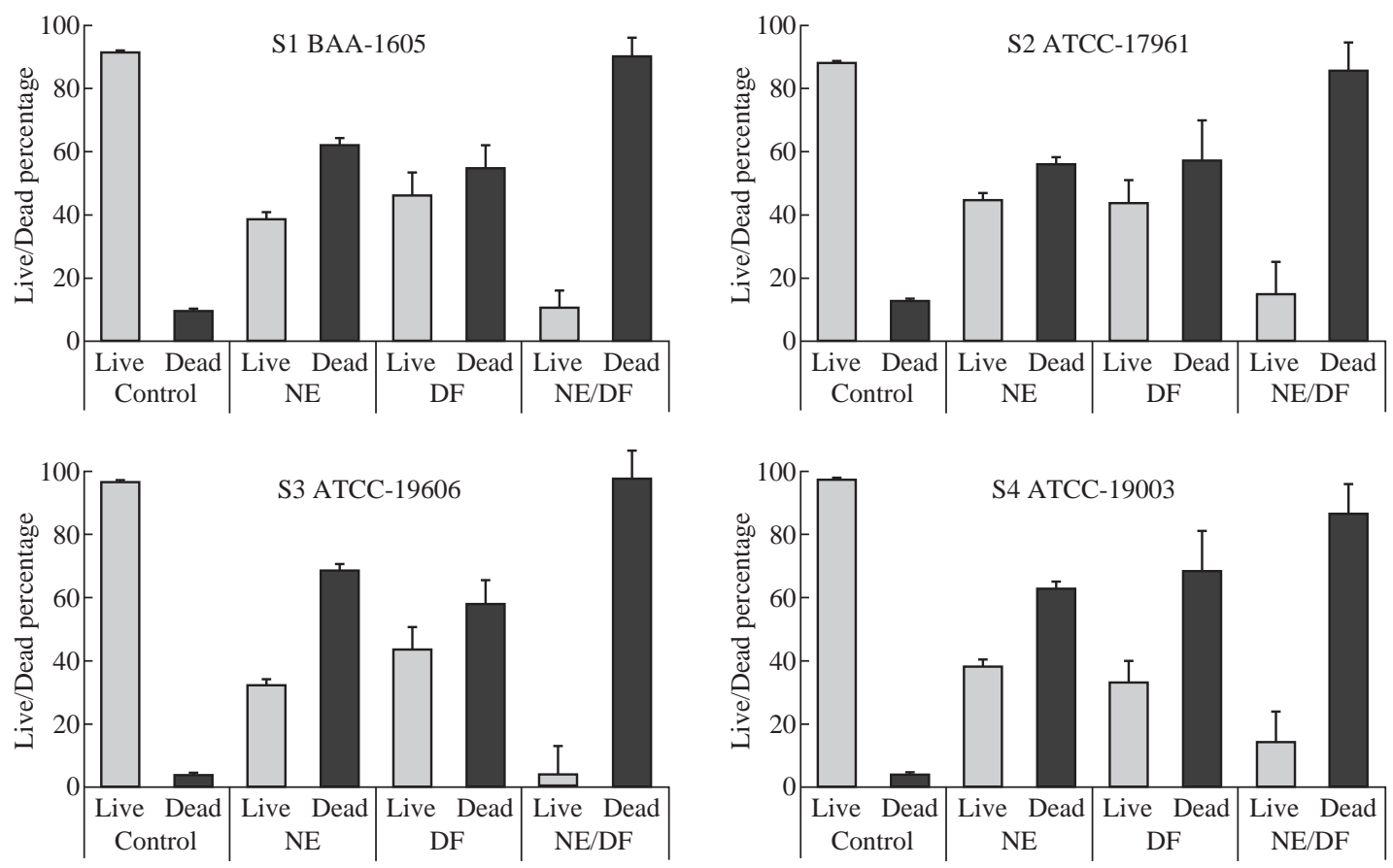

Fig. 4 Exposure of nanoemulsion (NE), deferiprone (DF), and nanoemulsion with deferiprone (NE/DF) to Acinetobacter baumannii biofilms. S1 BAA-1605; S2 ATCC-17961; S3 ATCC-19606; S4 ATCC-19003, and viability as measured with BacLight LIVE/DEAD stain.

condition. Propidium iodide was used to enumerate "dead" cells since it was a highly charged molecule and was unable to pass through cells having a strong electrochemical gradient across the membrane. The images reflected different green and red florescence intensities, and nanoemulsion with deferiprone (NE/ DF) showed a significantly increased dead cell area $(p<0.05)$. NE 250 dilution and $10 \mathrm{mM}$ concentration of DF showed decreased red color intensity when compared to combined NE/DF (250 dilution of NE with $10 \mathrm{mM}$ DF) and reduced green intensity with lower dilution NE and the higher concentration of DF. The level of dead cell intensity was $84.9 \%$ to $96.6 \%$ in nanoemulsion with deferiprone treated groups. NE alone inhibited biofilm formation of A. baumannii (31.7\% to $44.5 \%)$ and deferiprone alone inhibited formation from $53.9 \%$ to $67.6 \%$. The combined effect of NE and DF showed a 1.69-fold increase in killing effect. A higher level of green intensity (87.6\% to $96.8 \%)$ was observed in the negative control.

\section{Scanning electron microscopy (SEM) observation}

SEM examination was carried out to check for possible morphological changes in A. baumannii caused by treatment by nanoemulsion (NE), deferiprone (DF) and nanoemulsion with deferiprone (NE/DF). Significant morphological changes were observed in the cells treated with NE, DF and NE/ DF compared to pili-bonded intact control cells (Fig. 6). After NE, DF and NE/DF treatment, cell surfaces were remarkably disintegrated with highly reduced cell numbers. Irregular boundaries were observed with unclear cell walls and biofilms appeared to have collapsed, apparently a direct effect of exposure to NE, DF and NE/DF. The enlarged clear images of A. baumannii control biofilms showed (Fig. 7) the beautifully arranged and attached bacteria with pili structure needed for exceptional adherence and biofilm formation. No pili structure similar to that seen in the control was observed with any treated groups (NE, DF and NE/DF). We hypothesize that biofilm destruction starts from pili removal and is followed by membrane destruction.

\section{Activity of nanoemulsion and its components}

Effectiveness of nanoemulsion was greater than that of its components at $1 \mathrm{~h}$, with $10 \%$ Brij (26\% dead intensity), $25 \%$ soybean oil (19\% dead intensity), $60 \mathrm{mM}$ EDTA (24\% dead intensity) and all unemulsified ingredients (53\% dead intensity) showing lower activity. Compared to the control, nanoemulsion showed 91\% inhibition of A. baumannii (Fig. 8), indicating that nanoemulsion had the efficacy in excess of its ingredients. 

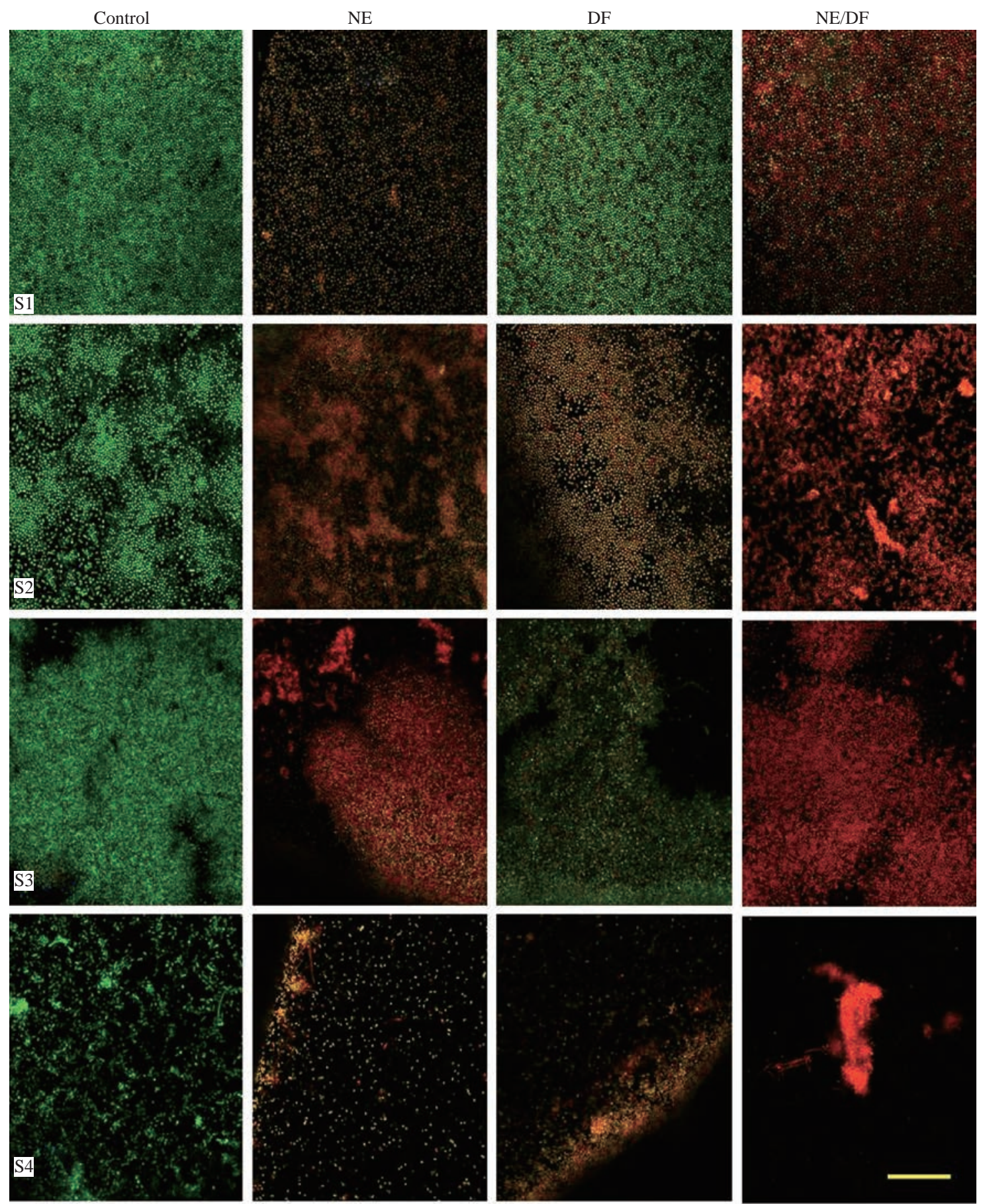

Fig. 5 Confocal micrographs of Acinetobacter baumannii biofilms. S1 BAA-1605; S2 ATCC-17961; S3 ATCC-19606; and S4 ATCC19003). Control (no treatment), DF (10 mM deferiprone), NE (Nanoemulsion, 250 dilution), NE/DF nanoemulsion with deferiprone (250 dilution with $10 \mathrm{mM})$. (Bar: $25 \mu \mathrm{m})$.

\section{Discussion}

Combining the new generation disinfectant, nanoemulsion (NE) [20], and the novel iron chelator, deferiprone (DF), is a sensible approach to the control of planktonic and biofilm infection by the MDRbacterial pathogen A. baumannii. Examination into the combination of NE with DF as an antibacterial remedy was impelled by the known issue of development of antimicrobial drug resistant strains. From time to time, there was inappropriate usage of currently available antibiotics, disinfectants and antiseptics due to extensively existence of multi drug resistance microorganism [21]. The above mentioned shortcomings rationalize to promote, explore and develop the novel antibacterial medications which aim precise pathogens target while being harmless for the 

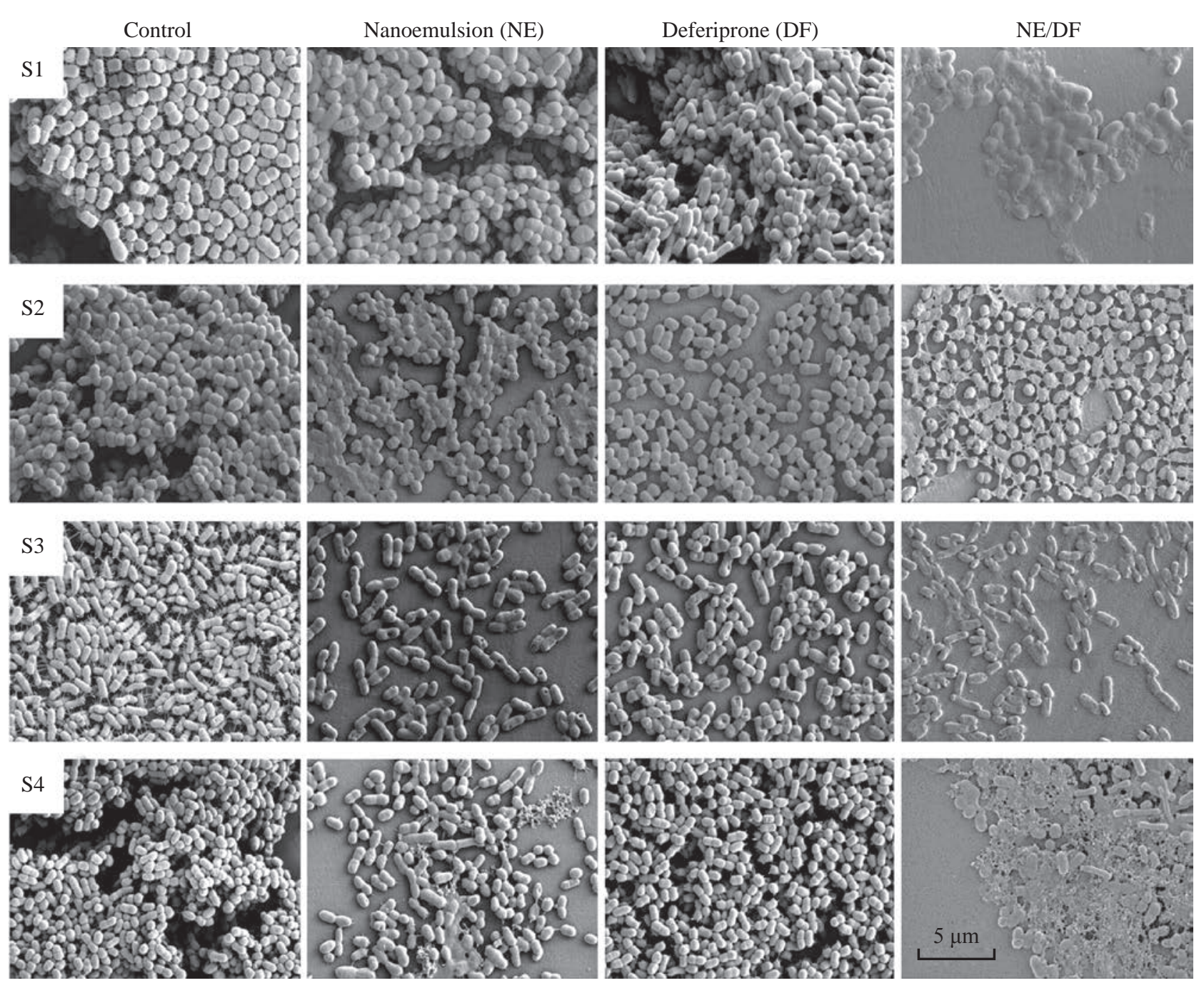

Fig. 6 Scanning electron microscope image of control (no treatment), DF treatment (10 mM deferiprone), NE treatment (Nanoemulsion, 250 dilution), and NE/DF treatment (nanoemulsion (250 dilution) with $10 \mathrm{mM}$ deferiprone) on Acinetobacter baumannii biofilms. S1 BAA-1605; S2 ATCC-17961; S3 ATCC-19606; and S4 ATCC-19003.
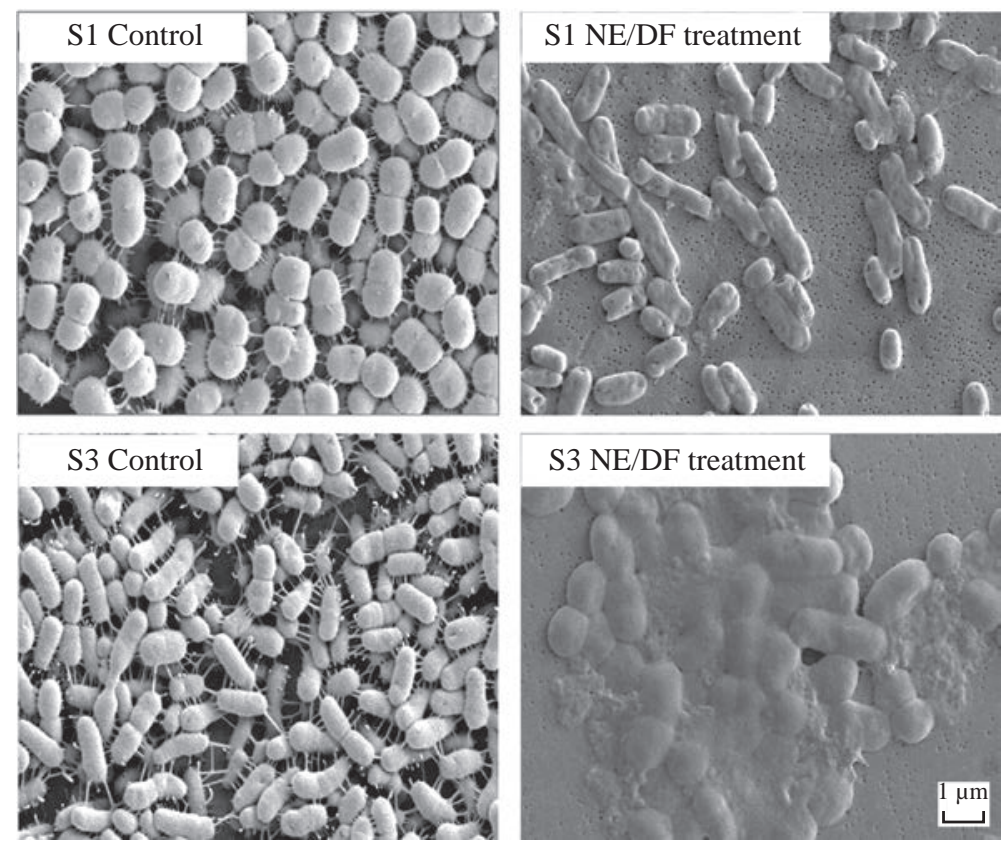

Fig. 7 Scanning electron microscope of control (no treatment), and NE/DF treatment shows mechanisms of pili and membrane disruption in Acinetobacter baumannii biofilms. S1 BAA-1605; S3 ATCC-19606. 

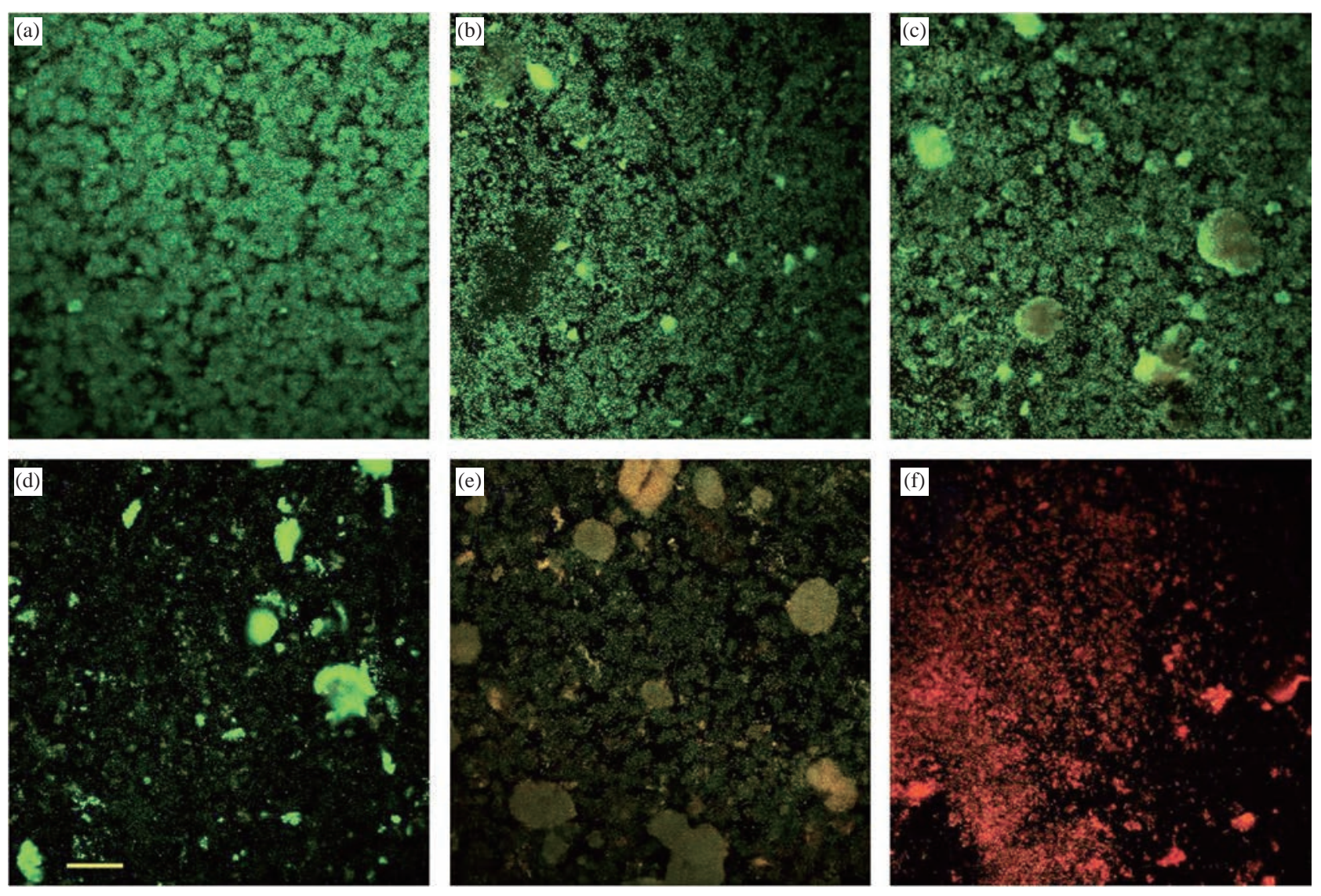

Fig. 8 LIVE/DEAD stained confocal micrographs shows Acinetobacter baumannii biofilm (BAA-1605) treated with nanoemulsion or with its ingredients. (a) Control (no treatment); (b) 25\% soybean oil; (c) $60 \mathrm{mM} \mathrm{EDTA;} \mathrm{(d)} \mathrm{Brij} \mathrm{30;} \mathrm{(e)} \mathrm{all} \mathrm{ingredients} \mathrm{without}$ emulsification; and (f) nanoemulsion treated (Bar: $50 \mu \mathrm{m})$.

infected community. Since NE mechanism targets only non-specific damage of microbial cell membranes, due to this property, NEs are improbable to make consequence to progress the drug resistant microbial communities. The iron chelation properties of DF can improve the antibacterial potential of NE, and metal chelators have already gone through FDA approvals, toxicity tests and preclinical animal testing, so this material might tender an unconventional treatment options. NEs have been reported to have extensive bactericidal, sporicidal and virucidal effects $[5,6$, 22]. NEs at biocidal concentrations are non-toxic in short term application to skin, mucous membranes, and the gastrointestinal tract [2]. Likewise, abundant investigations assessed the possible capability of iron chelators as remedial agents towards different microorganism among merely varied accomplishments [7-9], leading to the idea that the combination of $\mathrm{NE}$ and DF is a logical approach to developing new antimicrobials for MDR A. baumannii.

A. baumannii is exceptionally hard to treat for its nosocomial reach and infectivity in immunecompromised patients $[15,16]$. However, the applications of nanoemulsion with deferiprone (NE/ DF) to eradicate A. baumannii biofilms have not been investigated. This paper demonstrated that NE, DF and NE/DF formulations had inhibitory activity against A. baumannii adherence and biofilm formation. The results showed that NE/DF inhibited $A$. baumanii with MBC occurring at dilutions/concentration of S1 (320 dilutions/3.13 mM), S2 (213 dilution/ $5.21 \mathrm{mM}$ ), S3 (427 dilution/2.6 mM) and $\mathrm{S} 4$ (320 dilutions $/ 3.13 \mathrm{mM}$ ). Myc et al. [22] found that the active concentration of a different nanoemulsion never exceeded $0.1 \%$ of NE. Others have reported higher dilutions of NE with a higher activity $[1,2,21]$. Thompson et al. [23] found that DF showed inhibition against $A$. baumannii $(128 \mu \mathrm{g} / \mathrm{mL})$, P. aeruginosa, K. pneumonia and $E$. coli. Reports are also explaining DF has the capacity to redistrict the growth of S. aureus [7], and ironavailability reduces the growth of Y. enterocolitica [24]. In addition, septicemia associated mortality causes V. vulnificus inhibited by DF [25]. Furthermore, no reports are available for bacterial utilization of DF as iron supplement; hence, DF did not promote bacterial growth [7]. 
We were initially hindered in antimicrobial testing against $A$. baumannii because of its biofilm growth pattern in vitro. This bacterium is a strictly aerobic bacterium that does not form a contiguous cell layer even after extended incubation [26], and grows and forms a strong layer of biofilm only at the air-liquid interface. The strains had weak adhesion patterns to various materials, with the exception of glass [27]. We used a slanted glass coverslip $(20 \mathrm{~mm} \times 11 \mathrm{~mm})$ insert. Biofilm at the air-liquid interface then colonized the slanted coverslip, providing for enhanced biofilm growth and better experimental handling for adherence assay, and for confocal and scanning electron microscopy observations.

The inhibitory effect of NE on adherence of cells to glass surfaces indicated that NE, DF and the combined form of nanoemulsion with deferiprone (NE/DF) significantly inhibited planktonic A. baumannii growth and stopped the adherence to glass surfaces. The combined form of NE/DF showed higher adherence inhibition compared to the individual ingredients. The lower dilution of NE (1000 dilution) and higher concentration of DF (2 mM) showed higher inhibition of adhesion. NE adherence inhibition results supported earlier works related to antibacterial properties of micro-emulsions towards planktonic bacteria and also biofilm stage [28]. In addition to their comprehensible anti-planktonic properties, it is now likely to propose that nanoemulsions are greatly effectual against microbial biofilm. Relatively higher concentrations of deferiprone ( 0.5 to $2.0 \mathrm{mM}$ ) inhibited the adherence of A. baumannii and similar results were observed by Kim and Shin [7] in Yersinia enterocolitica, S. aureus and Vibrio species. Deferiprone of higher concentrations lead to a severely iron-limited growth environment for bacteria resulted problem to survive $[9,30]$.

Confocal laser scanning microscopy (CLSM) has been widely used to study biofilm structure, composition and metabolism in several different microorganisms [29]. A big advantage of CLSM is that it allows in-depth analysis of biological structures, without killing or damaging the biological structure [18]. LIVE/DEAD staining has been used as an indicator of cell viability, as determined by the integrity of the cell wall membrane in many bacterial populations, including biofilms [30]. Biofilms treated with NE, DF and NE/DF showed marked dead cells at $250 \mathrm{NE}$ dilutions, $10 \mathrm{mM} \mathrm{DF}$ and combined NE/ DF, which was confirmed by bacterial viability staining. Moreover, from LIVE/DEAD staining it was found that nanoemulsion with deferiprone (NE/DF) caused significant membrane damage to bacteria. Our results of biofilms disruption by NE were synchronized with previously results of AlAdham et al. [28] for P. aeruginosa and S. aureus by microemulsion. In addition, our previous work indicated that nanoemulsion had a strong effect on controlling cariogenic plaque formation and resulting dental caries [1]. The processes of pathogenic bacteria multiplication and immune function require iron as a growth-essential nutrient, and the conditions that limits its presence will affect the growth of bacteria. Deferiprone leads to a severely iron-limited growth environment and chelation of iron from bacteria. Iron chelation may destabilize and mediate osmotic damage to the bacterial membrane [31].

Extracellular fibers are vital to the persistence of numerous human pathogenic microbes. Weakening pili destruction is a suitable strategy to fight bacterial pathogenesis whilst maintaining host defenses and host flora. Targeting organism-specific virulence factors has turned out to be a lucrative approach in the development of new antimicrobial therapies and might lessen the opportunities for the resistance mechanisms emergence when compared to their bactericidal equivalents [32]. A huge number of Gramnegative pathogens use the chaperone-usher pathway for the adhesive fiber assembly which play a role in host-pathogen interactions that are significant for infections [33]. The present study clearly demonstrated the disruption and removal of A. baumannii pili upon treatment by NE, DF and NE/DF. Complete disappearance of pili structure was observed with NE/ DF treatment (Fig. 8(a)). NE, DF and NE/DF treatment led to cell surfaces that were extremely disintegrated, without clear cell wall margins. This might reflect cell wall disruption that lead to non-viability and biofilm destruction. One such study was reported for a NE against Candida albicans [22]. Certain reports stated that biofilm microbes were extremely resistant to common cleaning agents, but were susceptible to NEs [34]. Deferiprone is also known to assist in its movement through the host cell membranes and possibly through bacterial membranes as well. Hence, the free iron may be chelated both outside and inside the bacteria, thus elucidating the improved efficiency of deferiprone [23].

The inventor of the nanoemulsion [35] proposed that during the preparation of nanoemulsions under high shear forces in a microfluidizer, that shear energy was 
stored in the oil droplets, giving them high energy. He further proposed that this energy was passed to bacteria upon fusion of the droplets with the bacteria, disrupting the bacterial membrane. Therefore, NE would be attracted by the pathogens due to surface charge of nano-droplet, once droplet struck the pathogens with high pressure (nano-droplet produced at very high pressure, 20,000 psi) which would be released to cause disruption effect on the cell wall/membrane of pathogen. Further anti-biofilm ingredients also continued to do the anti-biofilm activity to control the infection. We demonstrated that the efficacy of NE was greater than that of its individual ingredients, indicating that nanoemulsion had the efficacy in excess of its individual ingredients [1]. These results supported the inventor's assertion.

In addition to non-specific disruption and high energy release $[2,3]$, the nanoemulsion contains the active agent EDTA. EDTA has two effects on bacterial cells: It causes an increase in permeability to substances that normally cannot enter [36] and, in addition, initiates the loss of a significant fraction of the surface lipopolysaccharide [37]. A synergistic relationship against a variety of organisms was demonstrated when EDTA was included with neomycin and with minocycline [38]. The authors noted that the combination of EDTA and antibiotic was much more effective than either ingredient alone. We propose that the loss of lipopolysaccharide in double-membraned, Gram-negative A. baumannii initiated by EDTA allows more complete disruption by nanoemulsion.

Deferiprone is a new synthetic iron-chelator and the most extensively studied iron chelator to date [39]. Clinical studies have demonstrated that 75 to $100 \mathrm{mg} / \mathrm{kg} /$ day of deferiprone [40] is safe to the host. Deferiprone creates strong complex with iron at physiological $\mathrm{pH}$, and this property has capacities to redistrict the growth of $Y$. enterocolitica [24], V. vulnificus [25], S. aureus [9], Rhizopus oryzae [40], and A. baumannii [39]. Therefore, we propose that iron depletion treatment may lead to restriction in the bacterial growth and immune functions of $A$. baumannii [31], because A. baumannii is associated with high iron (Fe III) content in its composition [39].

\section{Conclusions}

The combined form of nanoemulsion and deferiprone showed extraordinary inhibition property on the increase of $A$. baumannii in low doses. NE/DF efficiently controlled glass surface adherence of $A$. baumannii and also consequently biofilm inhibitions. The inhibitory adherence, biofilm and morphological disruption with pili destruction tendencies of NE/ DF advocate that this substance possibly will be useful to develop potential antimicrobial drug for immuno-compromised patients, military combat field members injured in wars and also might be a perfect substance for instrument/surface decontamination of $A$. baumannii.

\section{Acknowledgements:}

Sincere appreciation is extended to the authorities of Naval Medical Research Unit San Antonio (NAMRUSA), San Antonio, TX and Science and Engineering Research Board (SERB), India (\# SERB/LS-267/2014) for providing fund and facilities to perform this research. We would also like to acknowledge Professor Ralph H. Rawls, Department of Comprehensive Dentistry, University of Texas Health at San Antonio, for his valuable guidance.

\section{Conflict of Interest}

The authors declare no conflict of interest pertaining to the publication of this research paper.

\section{References}

[1] K. Ramalingam, B.T. Amaechi, H.R. Rawls, et al., Antimicrobial activity of nanoemulsion on cariogenic planktonic and biofilm organisms. Arch Oral Biol, 2012, 57: $15-22$.

[2] T. Hamouda, M.M. Hayes, and Z. Cao, A novel surfactant nanoemulsion with broad-spectrum sporicidal activity against Bacillus spores. J Infect Dis., 1999, 180: 19391949.

[3] D.C. Wright, Antimicrobial oil-in-water emulsions. US patent 5,475,667, 1996.

[4] V.A. Lee, R. Karthikeyan, H.R. Rawls, et al., Anticariogenic effect of a cetylpyridinum chloride containing nanoemulsion. $J$ Dent, 2010, 38: 742-749.

[5] T. Hamouda, A. Myc, B. Donovan, et al., A novel surfactant nanoemulsion with a unique nonirritant topical antimicrobial activity against bacteria enveloped viruses and fungi. Microbiol Res, 2001, 156: 1-7.

[6] B.W. Donovan, J.D. Reuter, Z. Cao, et al., Prevention of murine influenza A virus pneumonitis by surfactant nanoemulsions. Antivir Chem Chemother, 2001, 11: 41-49.

[7] C.M. Kim, S.H. Shin, Effect of iron-chelator deferiprone on the in vitro growth of staphylococci. $J$ Korean Med Sci, 2009, 24: 289-295.

[8] S.S. Fernandes, A. Nunes, A.R. Gomes, et al., Identification of a new hexadentate iron chelator 
capable of restricting the intra macrophagic growth of Mycobacterium avium. Microbes Infect., 2010, 12: 287294.

[9] Z. Debebe, T. Ammosova, D. Breuer, et al., Iron chelators of the di-2-pyridylketone thiosemicarbazone and 2-benzoylpyridine thiosemicarbazone series inhibit HIV1 transcription: identification of novel cellular targetsiron, cyclin-dependent kinase (CDK) 2, and CDK9. Mol Pharmacol., 2011, 79: 185-196.

[10] P. Chakrabarty, S. Rudra, M.A. Hossain, et al., Iron chelation therapy and thalassemia - an overview. Mymensingh Med J., 2011, 20: 513-519.

[11] H. Heli, S. Mirtorabi, K. Karimian, Advances in iron chelation: An update. Expert Opin Ther Pat., 2011, 21: 819-856.

[12] H.W. Boucher, G.H. Talbot, J.S. Bradley, et al., Bad bugs, no drugs: no ESKAPE! An update from the Infectious Diseases Society of America. Clin Infect Dis., 2009, 48: $1-12$.

[13] J.F. Turton, M.E. Kaufmann, M. Warner et al., A prevalent, multiresistant clone of Acinetobacter baumannii in Southeast England. J Hosp Infect., 2004, 58: 170-179.

[14] K.J. Towner, Acinetobacter: An old friend, but a new enemy. J Hosp Infect., 2009, 73: 355-363.

[15] J. Vila, J. Pachon, Therapeutic options for Acinetobacter baumannii infections. Expert Opin Pharmacother., 2008, 9: 587-599.

[16] A.P. Zavascki, C.G. Carvalhaes, R.C. Picao et al., Multidrug-resistant Pseudomonas aeruginosa and Acinetobacter baumannii: resistance mechanisms and implications for therapy. Expert Rev Anti Infect Ther., 2010, 8: 71-93.

[17] T.L. Stuart, M. Mulvey, A.E. Simor, et al., Acinetobacter baumannii in casualties returning from Afghanistan. Can J Infect Control, 2007, 22: 152-154.

[18] T.R. Neu, J.R. Lawrence, Development and structure of microbial stream biofilms as studied by confocal laser scanning microscopy. FEMS Microb Ecol., 1997, 24: 1125.

[19] J.C. Araujo, F.C. Teran, R.A. Oliveira, et al., Comparison of hexamethyldisilazane and critical point drying treatments for SEM analysis of anaerobic biofilms and granular sludge. $J$ Electron Microsc (Tokyo), 2003, 52: 429-433.

[20] M.J. Lawrence, G.D. Rees, Microemulsion-based media as novel drug delivery systems. Adv Drug Deliv Rev., 2000, 45: 89-121.

[21] P.C. Teixeira, G.M. Leite, R.J. Domingues, et al., Antimicrobial effects of a microemulsion and a nanoemulsion on enteric and other pathogens and biofilms. Int J Food Microbiol., 2007, 118: 15-19.

[22] A. Myc, T. Vanhecke, J.L. Landers et al., The fungicidal activity of novel nanoemulsion (X8W60PC) against clinically important yeast and filamentous fungi. Mycopathologia, 2001, 155: 195-201.

[23] M.G. Thompson, B.W. Corey, Y. Si, et al., Antibacterial activities of iron chelators against common nosocomial pathogens. Antimicrob Agents Chemother., 2012, 56(10): $5419-5421$.

[24] B. Lesic, J. Foulon, E. Carniel, Comparison of the effects of deferiproneversus deferoxamine on growth and virulence of Yersinia enterocolitica. Antimicrob Agents Chemother., 2002, 46: 1741-1745.
[25] C.M. Kim, R.Y. Park, M.H. Choi et al., Ferrophilic characteristics of Vibrio vulnificus and potential usefulness of iron chelatingtherapy. J Infect Dis., 2007, 195: 90-98.

[26] A. Tomaras, C. Dorsey, R. Edelmann, et al., Attachment to and biofilm formation on abiotic surfaces by Acinetobacter baumannii: Involvement of a novel chaperone-usher pili assembly system. Microbiology, 2003, 149: 3473-3484.

[27] G.F.M. Moura Costa, M.C.B. Tognim, C.L. Cardoso, et al., Preliminary evaluation of adherence on abiotic and cellular surfaces of Acinetobacter baumannii strains isolated from catheter tips. Braz J Infect Dis., 2006, 10: 346-351.

[28] I.S.I. Al-Adham, E. Khalil, N.D. Al-Hmoud, et al., Microemulsions are membrane-active, antimicrobial, self preserving systems. J Appl Microbiol., 2000, 89: 32-39.

[29] A.M. Bodor, L. Jansch, J. Wissing, et al., The luxS mutation causes loosely-bound biofilms in Shewanella oneidensis. BMC Res Notes, 2011, 4: 180.

[30] K. Flemming, C. Klingenberg, J.P. Cavanagh, et al., High in vitro antimicrobial activity of synthetic antimicrobial peptidomimetics against staphylococcal biofilms. $J$ Antimicrob Chemother., 2009, 63: 136-145.

[31] R.T. Ellison 3rd, T.J. Giehl, Killing of Gram-negative bacteria by lactoferrin and lysozyme. J Clin Invest, 1991, 88: 1080-1091.

[32] J.S. Pinkner, H. Remaut, F. Buelens, et al., Rationally designed small compounds inhibit pilus biogenesis in uropathogenic bacteria. Proc Natl Acad Sci USA, 2006, 103: 17897-17902.

[33] L.B. Purevdorj-Gage LB.P. Stoodley, Biofilm structure, behavior, and hydrodynamics. Microbial biofilms. ASM Press, 2004: 160-173.

[34] T. Moretro, S. Langsrud, Listeria monocytogenes: biofilm formation and persistence in food processing environments. Biofilms, 2004, 1: 107-121.

[35] J.R. Baker Jr, T. Hamouda, A. Shih, et al., Non-toxic antimicrobial compositions and methods of use. US Patent 6,635,676, 2003.

[36] H.L. Ennis HL Bacterial resistance to the synergistic antibiotics of the PA 114, streptogramin, and vernamycin complexes. J Bacteriol, 1967, 93: 1881-1887.

[37] L. Leive, Release of lipopolysaccharide by EDTA treatment of E. coli. Biochem Biophys Res Commun, 1965, 21: 290-296.

[38] I. Raad, I. Chatzinikolaou, G. Chaiban, In vitro and ex vivo activities of minocycline and EDTA against microorganisms embedded in biofilm on catheter surfaces. Antimicrob Agents Chemother, 2003, 47: 3580-3585.

[39] L. Leseleuc, G. Harris, R.K. Lee, et al., In vitro and in vivo biological activity of iron chelators and gallium nitrate against Acinetobacter baumannii. Antimicrob Agents Chemother, 2012, 56(10): 5397-5400.

[40] A.S. Ibrahim, T. Gebermariam, Y. Fu, et al., The iron chelator deferasirox protects mice from mucormycosis through iron starvation. J Clin Invest, 2007, 117: 26492657.

Copyright $\odot$ Karthikeyan Ramalingam, Valerie Lee. This is an open-access article distributed under the terms of the Creative Commons Attribution License, which permits unrestricted use, distribution, and reproduction in any medium, provided the original author and source are credited. 